\title{
The Ketogenic Diet: Proposed Mechanisms of Action
}

\author{
Kirk Nylen, Sergei Likhodii, and W. McIntyre Burnham \\ Department of Pharmacology and University of Toronto Epilepsy Research Program (UTERP), University of Toronto, Toronto, \\ Ontario M5S 1A8, Canada
}

Summary: The ketogenic diet is a high-fat, low-carbohydrate diet used to treat drug-resistant seizures, especially in children. A number of possible mechanisms of action have been proposed to explain the anticonvulsant effects of the diet. Four of these hypothetical mechanisms are discussed in the present article: the $\mathrm{pH}$ hypothesis, the metabolic hypotheses, the amino acid hypothesis, and the ketone hypothesis. Key Words: Epilepsy, ketogenic diet, pH hypothesis, metabolic hypothesis, amino acid hypothesis, ketone hypothesis.

\section{INTRODUCTION}

The ketogenic diet is a high-fat, low-carbohydrate diet used to treat drug-resistant seizures, especially in children. The ketogenic diet was first formulated by Wilder ${ }^{1}$ in 1921. It lost favor after the invention of phenytoin in 1938 , but came back into prominence in the 1990s when it became clear that the diet could control seizures that resist anticonvulsant drugs. ${ }^{2}$

Over the years, a number of hypotheses have been offered to explain the ketogenic diet's anticonvulsant actions. These include: the $\mathrm{pH}$ hypothesis, the metabolic hypotheses, the amino acid hypotheses (including the GABA shunt hypothesis), the ketone theories (including the acetone hypothesis), the caloric restriction hypothesis, the brain lipids hypothesis, and the noradrenaline hypothesis. ${ }^{3}$ The present discussion will briefly consider the first four of these. A more complete discussion of these and the other hypotheses can be found in a useful book, entitled Epilepsy and the Ketogenic Diet, ${ }^{3}$ and in a useful review article, entitled The Neuropharmacology of the Ketogenic Diet. ${ }^{4}$

\section{THE pH HYPOTHESIS}

The $\mathrm{pH}$ hypothesis was proposed by Bridge and $\operatorname{Iob}^{5}$ in 1931. The gist of this hypothesis is the idea that the

Address correspondence and reprint requests to: W. McIntyre Burnham, Department of Pharmacology, Medical Sciences Building, 1 King's College Circle, Toronto, Ontario M5S 1A8, Canada. E-mail: mac.burnham@utoronto.ca. ketogenic diet makes the blood (and brain) slightly acidic, and that this change in $\mathrm{pH}$ stops seizures. This hypothesis is based on the fact that in patients on the ketogenic diet (in the near absence of carbohydrates) fats are used as a major source of energy. As the fats are metabolized by the liver, three fat-derived metabolites are formed: 1) acetoacetate, 2) beta-hydroxybutyrate, and 3) acetone. The first two of these compounds are keto acids, whereas only acetone is a true ketone. ${ }^{6}$ Traditionally, however, these three compounds are often referred to as the "ketone bodies."

Because acetoacetate and beta-hydroxybutyrate are ketoacids, Bridge and Iob $^{5}$ postulated that they might make the blood, and presumably the brain, slightly acidic. It has long been believed that that mild acidosis is anticonvulsant, whereas mild alkalosis is proconvulsant. Low $\mathrm{pH}$, for instance, has been shown to inhibit $\mathrm{pH}$ sensitive NMDA-type glutamate receptors and $\mathrm{pH}$-sensitive gap junctions, causing a possible decrease in neural excitation. ${ }^{7,8}$ Making the blood slightly acidic has also been offered as an explanation of how the carbonic anhydrase inhibitors, like acetazolamide, act to stop seizures. Bridge and $\mathrm{Iob}^{5}$ proposed a similar mechanism for the ketogenic diet.

The $\mathrm{pH}$ hypothesis was simple and very testable. Unfortunately, tests did not bear it out. In clinical studies, Huttenlocher ${ }^{9}$ showed that the ketogenic diet did not make the blood acidic, and Al-Mudallal et al. ${ }^{10}$ later showed in animal studies that the ketogenic does not change the $\mathrm{pH}$ of either the blood or the brain. 


\section{THE METABOLIC HYPOTHESES}

Another set of hypotheses may be called the "metabolic" hypotheses. There have been several of these. The present discussion will focus on three of them. The gist of all of these hypotheses is that the ketogenic diet makes the brain switch from a glucose-based metabolism to a ketone-based metabolism, and that this change somehow produces anticonvulsant effects.

\section{THE INCREASED BRAIN ENERGY HYPOTHESIS}

Perhaps the first researchers to suggest a "metabolic" hypothesis was DeVivo (i.e., Appleton and DeVivo" and Nordi and DeVivo ${ }^{12}$ ). The gist of his hypothesis was that the ketogenic diet provides the brain with extra energy.

DeVivo pointed out that the ketone bodies are a more efficient source of energy than glucose, because they produce more ATP per unit. ${ }^{11,12}$ That would mean that the brain of a person on the ketogenic diet would have more available energy (i.e., more ATP). That increase in available energy was postulated to have anticonvulsant effects. ${ }^{12}$ A corollary to this hypothesis was the hypothesis that the epileptic brain is deficient in energy, and that it is this deficiency that leads to seizures. DeVivo, ${ }^{12}$ in fact, makes this suggestion.

A question to be resolved regarding this hypothesis, and other metabolic hypotheses, is whether the epileptic brain is actually energy deficient. This has yet to be established. It should also be kept in mind that the metabolic shift in patients on the ketogenic diet is not complete. Glucose is still present in the blood of patients on the ketogenic diet, usually in the low normal range. ${ }^{9,13}$ This glucose is presumably entering into the brain and being used for energy. Even after some weeks of complete starvation, fats provide only about $60 \%$ of the brain's energy supply. ${ }^{14}$

\section{INCREASE IN MITOCHONDRIAL NUMBER}

A recent report that seems to complement the DeVivo hypothesis relates to the effects of the ketogenic diet on mitochondria. Bough et al. ${ }^{15}$ have reported that rats on the ketogenic diet produce more mitochondria than rats on normal rodent chow. Presumably, more mitochondria would also mean more energy for the brain, and, therefore, possibly, fewer seizures. As with the "increased brain energy" hypothesis, the question remains as to whether the epileptic brain is energy deficient, and whether supplying more energy will make the epileptic brain less excitable.

\section{"FAST" ENERGY AND THE KETOGENIC DIET}

A very different sort of metabolic hypothesis relates to the availability of "fast" energy. To simplify a rather complex discussion, the gist of this hypothesis is that glucose is necessary to provide the "fast" energy required for seizure activity and that in the absence of glucose, seizures can not occur. ${ }^{16,17}$

The idea behind this hypothesis is that the glucose found in people on a normal diet produces rapidly available, or "fast," energy through glycolysis, as well as less rapidly available, or "slow," energy through the Krebs cycle. Due to the easy availability of glucose in nondiet patients, seizures, which require "fast" energy, can occur. However, ketone bodies (present in people on a ketogenic diet) do not undergo glycolysis. Therefore, they can only provide "slow" energy via the Krebs cycle. Patients on the ketogenic diet, therefore, do not seize.

This hypothesis must account somehow for the fact that glucose is present at low-normal levels in patients on the diet, ${ }^{9,13}$ and also for the fact that hypoglycemia causes seizures.

It should be noted that that lowered levels of glucose in this hypothesis are thought to correlate with elevated levels of ketone bodies. This hypothesis, therefore, is compatible with the ketone hypotheses discussed below.

Several recent studies do seem to be in general agreement with the idea that decreasing glucose metabolism has anticonvulsant effects. Administration of fructose1,6-bisphosphate and 2-deoxyglucose have both shown anticonvulsant effects in acute animal seizure models, ${ }^{18}$ and 2-deoxyglucose has been shown to raise seizure thresholds and slow the rate of seizure development in kindled rats. ${ }^{19}$ Likewise, in clinical studies, a low gylcemic index diet, restricted to foods that produce little increase in the levels of blood glucose, has anticonvulsant actions, although that diet increases ketone bodies, as will as limiting glucose. ${ }^{20}$

\section{THE AMINO ACID THEORIES/THE GABA SHUNT HYPOTHESIS}

Another group of hypotheses relate to the amino acid transmitters. The "GABA shunt" hypothesis is one of these. The gist of the amino acid hypotheses is that the ketogenic diet modifies the balance of the amino acid transmitters in the brain in such a way as to suppress seizure activity. ${ }^{21}$ The GABA shunt hypothesis, in particular, suggests that the diet suppresses seizures by enhancing GABAergic inhibition, like many of the antiseizure drugs.

In a sense, the amino acid hypotheses are an offshoot of the metabolic hypotheses, since glutamate and GABA are produced by a "shunt" off the Krebs cycle. Instead of 
going from alpha-ketoglutarate to succinyl coenzyme A to succinate in the cycle (i.e., the direct route), the GABA shunt follows an indirect route, going from alpha-ketoglutarate to glutamate, and then to GABA and succinyl semialdehyde, before proceeding to succinate. ${ }^{22}$ The GABA shunt thus allows for the production of both of the major amino acid transmitters during the course of energy production. The GABA shunt hypothesis suggests that in the presence of the plentiful acetyl-CoA derived from fat metabolism, there is an increased production of alpha-ketoglutarate, and that this leads in turn to an increase in the production of glutamate, and especially GABA. $^{21}$

Questions related to this hypothesis are why GABA is increased more than glutamate, and also why enhancement of GABA by the diet suppresses seizures in patients in which GABA enhancement by the anti-seizure drugs has already failed. Finally, it must be noted that brain levels of GABA have been measured in animals on a ketogenic diet, and widespread elevations have not been found, although studies are continuing and there is a possibility that there may be regional changes occurring in discrete areas. ${ }^{4,11}$

\section{THE KETONE HYPOTHESES/THE ACETONE HYPOTHESIS}

A final group of hypotheses are the "ketone" hypotheses. The gist of these hypotheses is the idea that the ketone bodies themselves have direct anticonvulsant effects on the brain. The "acetone" hypothesis, in particular, suggests that it is the increase in blood and brain levels of acetone that causes the anticonvulsant effects of the diet. This hypothesis has been associated with our own research group in Toronto.

A ketone hypothesis was actually one of the earliest suggestions as to how the ketogenic diet might work. It was first suggested by Wilder ${ }^{1}$ when he introduced the diet, although he speculated that the active, anticonvulsant compound was acetoacetate.

Our own group has recently tested the effects of the ketone bodies in animal seizure models. In our hands, neither acetoacetate nor beta-hydroxybutyrate has shown anticonvulsant effects, ${ }^{23}$ although see Rho et al. ${ }^{24}$ Acetone, however, as with ketogenic diet itself, has broad spectrum anticonvulsant effects. ${ }^{23}$ It is effective against a variety of different seizure types and its anti-seizure effects are associated with little toxicity. ${ }^{23}$ In animals, these effects occur at blood acetone concentrations from 1 to $30 \mathrm{mM}$.

A question that must be addressed by the acetone hypothesis is whether the ketogenic diet elevates acetone to anticonvulsant levels in humans, and whether these acetone levels correlate with the diet's anticonvulsant effects. Preliminary data suggest acetone levels in pa- tients on the diet rise as high as $8 \mathrm{mM},{ }^{25}$ which would be in the anticonvulsant range in animals. As yet, however, clinical data showing a significant correlation between seizure control and blood levels of ketones/ketoacids are largely lacking.

\section{CONCLUSIONS}

In summary, a number of different hypotheses related to the mechanism of action of the ketogenic diet have been framed. No one currently champions the $\mathrm{pH}$ hypothesis, but all of the other hypotheses have supporters. The ketogenic diet remains a rich field for research.

Acknowledgments: This research done by our group on the acetone hypothesis was supported by funds from the Canadian Institutes of Health Science, the Savoy Foundation, the National Science and Engineering Council of Canada, and the Michael Bahen Chair of Epilepsy Research, held by W. M. Burnham. The authors would like to thank Heidi Wong, Warda Fatima, Yerusha Nuh, Osman Atkar, and Jerome Cheng for their help in preparation of the manuscript. The authors confirm that they have read the position of this journal on issues involved in ethical publication, and affirm that this report is consistent with those guidelines. The authors have no conflicts of interest to disclose. Dr. Nylen's present address is: Cancer Quality Council of Ontario Secretariat, Cancer Care Ontario, 620 University Avenue, Toronto, Ontario Canada M5G 2L7. Dr. Likhodii's present address is: Children's Hospital of Eastern Ontario, 401 Smyth Rd., Ottawa, Ontario, Canada K1H $8 \mathrm{~L} 1$.

\section{REFERENCES}

1. Wilder RM. The effects of ketonemia on the course of epilepsy. Mayo Clin Bull 1921;2:307-308.

2. Bailey AE, Pfeifer HH, Thiele EA. The use of diet in the treatment of epilepsy. Epilepsy Behav 2005;6:4-8.

3. Statstrom CE, Rho JM, eds. Epilepsy and Ketogenic Diet, 1 st ed. Totowa (NJ): Humana Press 2004.

4. Hartman AL, Gasior M, Vining EPG, Rogawski MA. The neuropharmacology of the ketogenic diet. Pediatr Neurol 2007;36:281292.

5. Bridge EM, Iob LV. The mechanism of the ketogenic diet in epilepsy. Johns Hopkins Med J 1931;48:373-389.

6. Likhodii SS, Burnham WM. The effects of ketone bodies on neuronal excitability. In: Stafstrom CE, Rho JM, eds. Epilepsy and the ketogenic diet, 1st ed. Totowa (NJ): Humana Press; 2004:217-228.

7. Schwartzkroin PA. Mechanisms underlying the anti-epileptic efficacy of ketogenic diet. Epilepsy Res 1999;37:171-180.

8. Perez Velazquez JL, Carlen PL. Gap junctions, synchrony and seizures. Trends Neurosci 2000;23:68-74.

9. Huttenlocher PR. Ketonemia and seizures: metabolic and anticonvulsant effects of two ketogenic diets in childhood epilepsy. Pediatr Res 1976;10:536-540.

10. Al-Mudallal AS, LaManna JC, Lust WD, Harik SI. Diet-induced ketosis does not cause cerebral acidosis. Epilepsia 1996;37:258261.

11. Appleton DB, DeVivo DC. An animal model for the ketogenic diet. Epilepsia 1974;15:211-227.

12. Nordi DR, De Vivo DC. Effects of the ketogenic diet on cerebral energy metabolism. In: Stafstrom CE, Rho JM, eds. Epilepsy and the ketogenic diet, 1st ed. Totowa (NJ): Humana Press; 2004:179184.

13. Vining EPG. Clinical efficacy of the ketogenic diet. Epilepsy Res 1999;37:181-190. 
14. Nehlig A. Brain uptake and metabolism of ketone bodies in animal models. Prostaglandins Leukot Essent Fatty Acids 2004;70:265275.

15. Bough KJ, Wetherington J, Hassel B, et al. Mitochondrial biogenesis in the anticonvulsant mechanism of the ketogenic diet. Ann Neurol 2006;60:223-235.

16. Greene AE, Todorova MT, Seyfried TN. Perspectives on the metabolic management of epilepsy through dietary reduction of glucose and elevation of ketone bodies. J Neurochem 2003;86:529537.

17. Seyfried TN, Greene AE, Todorova MT. Caloric restriction and epilepsy: historical perspectives, relationship to the ketogenic diet, and analysis in epileptic EL mice. In: Stafstrom CE, Rho JM, eds. Epilepsy and the ketogenic diet, 1st ed. Totowa (NJ): Humana Press, 2004:247-264.

18. Lian X, Khan FA, Stringer JL. Fructose-1,6-bisphosphate has anticonvulsant activity in models of acute seizures in adult rats. J Neurosci 2007;27:1207-1211.

19. Garriga-Canut M, Schoenke B, Qazi R, et al. 2-deoxyglucose reduces epilepsy progression by NRSF-CtBP-dependent meta- bolic regulation of chromatin structure. Nature Neurosci 2006;9:1382-1387.

20. Pfeifer HH, Thiele EA. Low-glycemic index treatment: a liberalized ketogenic diet for treatment of intractable epilepsy. Neurology 2005;65:1810-1812.

21. Yudkoff M, Daikhin Y, Nissim I, Nissim I. The ketogenic diet: interactions with brain amino acid handling. In: Stafstrom CE, Rho JM, eds. Epilepsy and the ketogenic diet, 1st ed. Totowa (NJ): Humana Press, 2004:185-200.

22. Lodish H, Berk A, Matsudaira P, et al. Molecular cell biology, 5th ed. New York: W.H. Freeman, 2004.

23. Likhodii SS, Serbanescu I, Cortez MA, Murphy P, Snead OC III, Burnham WM. Anticonvulsant properties of acetone, a brain ketone elevated by the ketogenic diet. Ann Neurol 2003;54:219-226.

24. Rho JM, Anderson GD, Donevan SD, White HS. Acetoacetate, acetone, and dibenzylamine (a contaminant in L- $(+)-\beta$-hydroxybutyrate) exhibit direct anticonvulsant actions in vivo. Epilepsia 2002;43:358-361.

25. Musa-Veloso K. Non-invasive detection of ketosis and its application in refractory epilepsy. Prostaglandins Leukot Essent Fatty Acids 2004;70:329-335. 